\title{
Sleep quality and daytime function in adults with cystic fibrosis and severe lung disease
}

\author{
D.R. Dancey*, E.D. Tullis*, R. Heslegrave ${ }^{\#}$, K. Thornley*, P.J. Hanly*
}

Sleep quality and daytime function in adults with cystic fibrosis and severe lung disease. D.R. Dancey, E.D. Tullis, R. Heslegrave, K. Thornley, P.J. Hanly. (C) ERS Journals Ltd 2002.

ABSTRACT: It was hypothesized that adult cystic fibrosis (CF) patients with severe lung disease have impaired daytime function related to nocturnal hypoxaemia and sleep disruption.

Nineteen CF patients (forced expiratory volume in one second $28 \pm 7 \%$ predicted) and 10 healthy subjects completed sleep diaries, overnight polysomnography (PSG), and assessment of daytime sleepiness and neurocognitive function.

CF patients tended to report more awakenings $\left(0.7 \pm 0.5\right.$ versus $\left.0.3 \pm 0.2 \cdot \mathrm{h}^{-1}, \mathrm{p}=0.08\right)$, and PSG revealed reduced sleep efficiency $(71 \pm 25$ versus $93 \pm 4 \%, p=0.004)$ and a higher frequency of awakenings (4.2 \pm 2.7 versus $\left.2.4 \pm 1.4 \cdot h^{-1}, p=0.06\right)$. Mean arterial oxygen saturation during sleep was lower in $C F$ patients $(84.4 \pm 6.8$ versus $94.3 \pm 1.5 \%$, $\mathbf{p}<0.0001$ ) and was associated with reduced sleep efficiency (regression coefficient $(\mathrm{r})=0.57, \mathrm{p}=0.014)$. $\mathrm{CF}$ patients had short sleep latency on the multiple sleep latency test $(6.7 \pm 3 \mathrm{~min})$. The $\mathrm{CF}$ group reported lower levels of activation and happiness and greater levels of fatigue $(p<0.01)$, which correlated with indices of sleep loss, such as sleep efficiency $(\mathrm{r}=\mathbf{0 . 4 7}, \mathrm{p}=\mathbf{0 . 0 5})$. Objective neurocognitive performance was also impaired in $\mathrm{CF}$ patients, reflected by lower throughput for simple addition/subtraction, serial reaction and colour-word conflict.

The authors concluded that adult cystic fibrosis patients with severe lung disease have impaired neurocognitive function and daytime sleepiness, which is partly related to chronic sleep loss and nocturnal hypoxaemia.

Eur Respir J 2002; 19: 504-510.
*St. Michael's Hospital, Dept of Medicine and "Dept of Psychiatry, University of Toronto, Toronto, ON, Canada.

Correspondence: P.J. Hanly

St. Michael's Hospital

30 Bond Street

Suite 6-049

Toronto

ON, Canada

MSB 1 W8.

Fax: 4168645922

E-mail: hanlyp@smh.toronto.on.ca

Keywords: Daytime sleepiness

hypoxaemia

neurocognitive function

sleep

Received: October 122000

Accepted after revision October 25 2001

This study was supported by the Canadian Cystic Fibrosis Foundation
Cystic fibrosis $(\mathrm{CF})$ occurs in $\sim 1$ in 2,000 live births and is currently the most common, life-threatening, genetic disorder in Caucasians [1]. Although increasing numbers of patients are living well into adulthood, many develop severe respiratory impairment due to bronchiectasis and chronic airflow limitation. In addition to causing respiratory disability, progressive, underlying lung disease also predisposes these patients to hypoxaemia, which has been associated with both sleep disruption and impairment of daytime function in other models of chronic respiratory disease, such as chronic obstructive pulmonary disease (COPD) and obstructive sleep apnoea $[2,3]$. There have been no previous studies which have evaluated adult CF patients in this way. The objective of this study was to determine whether adult CF patients with severe lung disease have impaired daytime function, and whether this is related to sleep disruption and nocturnal hypoxaemia.

\section{Materials and methods}

\section{Study subjects}

Adult CF patients who had significant airflow limitation (forced expiratory volume in one second $(\mathrm{FEV} 1)<40 \%$ predicted) were recruited from the adult
CF Programme at St. Michael's Hospital. The diagnosis of CF had been confirmed by an abnormal sweat chloride test and/or the presence of two diseasecausing mutations. Subjects were required to be clinically stable for at least 1 month prior to the study (no hospital admissions or exacerbations of lung disease). They were excluded by the presence of primary cardiac disease, pulmonary disease unrelated to $\mathrm{CF}$, primary neurological, psychiatric or sleep disorders, home oxygen therapy, or medication within 2 weeks of the study, known to alter sleep or cognitive function. No caffeine or alcohol was consumed for $48 \mathrm{~h}$ prior to the study. Healthy, age-matched control subjects were enrolled in the same study protocol. They were recruited by advertising within the hospital for nonsmoking, nonsnoring volunteers who did not have a history of medical or sleep disorders, and were taking no medication. All applicants were assessed (P.J. Hanly) prior to enrolment to confirm that they had a regular sleep schedule and that they did not consume excessive quantities of alcohol or caffeine.

\section{Study design}

All participants completed a detailed sleep diary for 2 weeks prior to their overnight polysomnography 
(PSG) in the sleep laboratory. On the day following PSG, sleepiness, neurocognitive function and oxygen saturation during exercise (6-min walk test) were evaluated in all subjects. In addition, CF patients performed spirometry and arterial blood gases were measured. Informed consent was obtained and the study was approved by the hospital's Research Ethics Board.

\section{Overnight polysomnography}

All subjects underwent overnight PSG using a computerized acquisition system (Sandman; Mallinckrodt Nellcor Puritan Bennett (Melville), Ottawa, ON, Canada) at a speed of $10 \mathrm{~mm} \cdot \mathrm{s}^{-1}$. Surface electrodes were used to record two-channel electroencephalogram (EEG) $\left(\mathrm{C}_{3} \mathrm{~A}_{2}, \mathrm{C}_{4} \mathrm{~A}_{1}\right)$, electrooculogram $\left(\mathrm{F}_{7} \mathrm{~A}_{1}\right.$, $\mathrm{F}_{8} \mathrm{~A}_{2}$ ) and submental electromyogram (EMG). Airflow at the nose was monitored by a thermocouple sensor secured beneath the nares (Ultima airflow sensor; Braebon Medical Corporation, Kanata, ON, Canada). Respiratory effort was recorded by respiratory plethysmography, with transducers placed around the chest and abdomen (Respitrace; Ambulatory Monitoring, Ardsley, NY, USA). Arterial oxygen saturation $\left(\mathrm{Sa}, \mathrm{O}_{2}\right)$ was monitored using a pulse oximeter (Biox 3740; Ohmeda, Boulder, CO, USA). The partial pressure of $\mathrm{CO}_{2}$ was monitored transcutaneously $\left(P \mathrm{t}, \mathrm{CO}_{2}\right)$ using a $\mathrm{PCO}_{2}$ sensor placed on the anterior chest wall and attached to a $\mathrm{Pt}_{\mathrm{t}} \mathrm{CO}_{2}$ monitor (Micro Gas 7640; Kontron Instruments, Watford, UK). The $P \mathrm{t}, \mathrm{CO}_{2}$ was recorded continuously on a slow recorder (paper speed $20 \mathrm{~cm} \cdot \mathrm{h}^{-1}$ ), which was synchronized to the PSG. The electrocardiogram was recorded from standard limb leads. Leg movements were recorded from bilateral surface EMG electrodes placed over the anterior tibialis muscle.

Polysomnograms were scored manually. Sleep stage and arousals were determined according to established criteria [4]. An arousal was defined as an awakening from sleep lasting between 3-15 s, manifested by simultaneous alpha activity on the EEG, eye movements and EMG activation. If these changes persisted for $>15 \mathrm{~s}$, the PSG was scored as awake. An apnoea was defined as cessation of airflow for $>10 \mathrm{~s}$. A hypopnoea was defined as a reduction in the amplitude of respiratory effort by at least $50 \%$ of the sleeping baseline level for $>10 \mathrm{~s}$. The apnoea-hypopnoea index was defined as the number of apnoeas and hypopnoeas per hour of sleep. Periodic leg movements (PLM) were defined as four or more consecutive, involuntary leg movements during sleep, lasting 0.5-5.0 s, with an intermovement interval of 5-90 s. Mean $\mathrm{Sa}_{\mathrm{a}} \mathrm{O}_{2}$ during sleep was calculated by averaging the high and low $\mathrm{Sa}_{\mathrm{a}} \mathrm{O}_{2}$ for each 30 -s epoch. Mean $P \mathrm{t}, \mathrm{CO}_{2}$ during sleep was calculated from the average $P \mathrm{t}, \mathrm{CO}_{2}$ over 36-s intervals. Awake $\mathrm{Sa}_{\mathrm{a}} \mathrm{O}_{2}$ and $\mathrm{Pt}_{\mathrm{t}} \mathrm{CO}_{2}$ were similarly estimated by calculating mean $\mathrm{Sa}_{\mathrm{a}} \mathrm{O}_{2}$ and $P \mathrm{t}, \mathrm{CO}_{2}$ at rest, over $5 \mathrm{~min}$ of wakefulness at the beginning of the study.

\section{Daytime sleepiness}

Daytime sleepiness was assessed subjectively by the Epworth Sleepiness Scale (ESS) [5] and objectively by the Multiple Sleep Latency Test (MSLT) [6]. The ESS is a self-administered questionnaire, in which an individual rates, on a numerical scale, the likelihood of falling asleep under various circumstances, such as sitting in a car or watching television. The scale ranges from zero ("would never doze") to three ("high chance of dozing") and the potential score ranges from 0-24, with a higher score indicating increased perceived sleepiness. A score of $5.9 \pm 2.2$ has been reported in normal controls and $16 \pm 4.4$ in patients with severe obstructive sleep apnoea [5].

The MSLT was performed in a standardized fashion [6]. Each subject took four naps during the day at 09:00, 11:00, 13:00, and 15:00 h. Sleep onset was defined as the time from "lights out" to the first of three consecutive epochs of stage 1 nonrapid eye movement (NREM) sleep, or one epoch of any other sleep stage. Once sleep onset was identified, the participant was awakened in order to prevent consolidated sleep from improving their performance on subsequent naps. In addition, the patient was observed to ensure wakefulness was maintained between naps.

\section{Neurocognitive function}

Neurocognitive function was evaluated in subjects by measuring both performance (objective measure) and the participants' impression of their daytime function (subjective measure), using the Walter Reed Performance Assessment Battery (version 3.46, 1992). This is a computerized battery of cognitive function tests, originally developed in 1985 [7]. The objective measures of performance included five standardized 3-min neurocognitive tasks (Wilkinson 4-choice serial reaction task, Baddeley grammatical reasoning task, Mannikin spatial orientation task, serial addition and subtraction task, and the colour-word conflict task (Stroop)). These performance tasks were scored and analysed in terms of throughput (a measurement of accuracy/speed reflecting the number of correct responses $\left.\cdot \mathrm{min}^{-1}\right)$. The subjective measures tested the participants' impression of their mood (Mood Scale, based on the Profile of Mood States, assessing fatigue, happiness, activation, depression, anger and fear) and their sleepiness (Stanford Sleepiness Scale). Details of the individual tasks are reported by THORNE et al. [7]. The tasks were performed in a quiet environment on a 486 personal computer, prior to each MSLT nap during the daytime. Each participant had an initial trial run-through of the Walter Reed Performance Assessment Battery at 08:00 $\mathrm{h}$ on the day of their MSLT, which was not included in the data analysis.

\section{Pulmonary function}

Spirometry was performed according to American Thoracic Society guidelines [8]. Blood gases were 
obtained from seated patients, breathing room air, during quiet wakefulness. The 6-min walk test was supervised by a trained technician.

\section{Statistical analysis}

Physiological data are presented as mean \pm SD. Apart from the neurocognitive data, the Mann-Whitney test was used to analyse differences between groups, with a p-value $<0.05$ considered statistically significant, and the Spearman correlation analysis was used to assess the relationship between variables. The neurocognitive data were analysed using multivariate analysis of variance for repeated measures to determine Group, Trial and Group/Trial interactions. Significant effects from the multivariate analyses are reported using the univariate degrees of freedom.

\section{Results}

\section{Study participants}

Nineteen $\mathrm{CF}$ and 10 control subjects participated in the study. Four CF patients refused arterial blood gases, and one control subject did not have neurocognitive function tested. The CF group had a greater proportion of men, a lower body mass index (BMI) and severe airflow limitation, associated with mild-tomoderate hypoxaemia. The 6-min walk test distance, and minimum $\mathrm{Sa}_{\mathrm{a}} \mathrm{O}_{2}$ were significantly less in the $\mathrm{CF}$ group than in controls (table 1).

Table 1.-Demographic and pulmonary function data for cystic fibrosis (CF) patients and control subjects

\begin{tabular}{|c|c|c|}
\hline & $\mathrm{CF}(\mathrm{n}=19)$ & Control $(n=10)$ \\
\hline Age yrs & $30 \pm 6$ & $27 \pm 5$ \\
\hline Male/female & $15 / 4$ & $5 / 5$ \\
\hline BMI & $21.8 \pm 3.3^{\#}$ & $25.5 \pm 2.0$ \\
\hline FEV1 & & \\
\hline $\begin{array}{l}\mathrm{L} \\
\% \text { predicted normal }\end{array}$ & $\begin{array}{l}1.1 \pm 0.35 \\
28 \pm 7\end{array}$ & \\
\hline Arterial blood gases & & \\
\hline $\mathrm{pH}$ & $7.4 \pm 0.03$ & \\
\hline $\mathrm{Pa}, \mathrm{CO}_{2} \mathrm{mmHg}$ & $44.2 \pm 7.3$ & \\
\hline$P \mathrm{a}, \mathrm{O}_{2} \mathrm{mmHg}$ & $65.6 \pm 8.2$ & \\
\hline$\left[\mathrm{HCO}_{3}^{-}\right] \mathrm{mmol} \cdot \mathrm{L}^{-1}$ & $28.3 \pm 4.2$ & \\
\hline $\mathrm{S}_{\mathrm{a}, \mathrm{O}_{2}}$ & $92.1 \pm 3.4$ & \\
\hline $\mathrm{Sa}_{\mathrm{a}, \mathrm{O}_{2}}$ awake $\%$ & $87 \pm 6^{* * *}$ & $95 \pm 2$ \\
\hline $\begin{array}{l}P \mathrm{t}, \mathrm{CO}_{2} \text { awake } \mathrm{mmHg} \\
\text { 6-min walk }\end{array}$ & $41.5 \pm 7.1$ & $47.0 \pm 2.3$ \\
\hline Distance m & $407 \pm 109$ & $534 \pm 74$ \\
\hline Min. $S \mathrm{a}, \mathrm{O}_{2} \%$ & $86.6 \pm 8.0^{+}$ & $93.5 \pm 5.0$ \\
\hline
\end{tabular}

Data are presented as mean \pm SD unless otherwise stated. BMI: body mass index; FEV1: forced expiratory volume in one second; $\mathrm{Pa}, \mathrm{CO}_{2}$ : arterial carbon dioxide tension; $\mathrm{Pa}, \mathrm{O}_{2}$ : arterial oxygen tension; $S_{\mathrm{a}, \mathrm{O}_{2}}$ : arterial oxygen saturation; $\mathrm{Sa}_{\mathrm{a}} \mathrm{O}_{2}$ awake: arterial oxygen saturation taken by oximetry while supine before nighttime sleep; $P \mathrm{t}_{1} \mathrm{CO}_{2}$ : transcutaneous carbon dioxide tension taken supine before nighttime sleep; Min.: minimum. \#: $\mathrm{p}=0.006 ;{ }^{\top} \mathrm{p}=0.012 ; * * *: \mathrm{p}<0.001$; ${ }^{+}$: $\mathrm{p}=0.008$ versus control.
Table 2. - Sleep diary data for cystic fibrosis (CF) patients and control subjects

\begin{tabular}{lcc}
\hline & $\mathrm{CF}(\mathrm{n}=15)$ & Control $(\mathrm{n}=8)$ \\
\hline Diary report days & $12.3 \pm 2.4$ & $13.9 \pm 0.4$ \\
Awakenings $\cdot \mathrm{h}^{-1}$ & $0.7 \pm 0.5^{\#}$ & $0.3 \pm 0.2$ \\
TST h & $8.1 \pm 1.5$ & $7.3 \pm 0.7$ \\
Caffeine drinks $\cdot$ day $^{-1}$ & $1.5 \pm 0.9$ & $1.3 \pm 0.9$ \\
Alcohol drinks $\cdot$ day $^{-1}$ & $0.3 \pm 0.5$ & $0.3 \pm 0.7$ \\
\hline
\end{tabular}

Data are presented as mean \pm SD. TST: estimated total sleep time. ${ }^{\#}$ : $\mathrm{p}=0.08$ versus control.

\section{Sleep diaries}

The CF group reported more nocturnal awakenings than controls, although this did not reach statistical significance (table 2). There was no difference between groups in the reported consumption of alcohol and caffeine and the estimation of total sleep time (TST).

\section{Polysomnography}

CF patients had significantly reduced TST and sleep efficiency, which was mainly due to prolonged wakefulness after sleep onset (WASO) (table 3). Although CF patients tended to have longer sleep latency, a higher proportion of stage one NREM sleep and a higher frequency of movement arousals and awakenings, these differences did not reach statistical significance. The frequency of PLM was within normal limits and was not significantly different between groups. The apnoea-hypopnoea index was normal in both groups (table 4). However, CF patients experienced significant hypoxaemia during sleep without evidence of significant hypoventilation. Furthermore, hypoxaemia was associated with reduced sleep efficiency (fig. 1).

Table 3. - Polysomnographic data for cystic fibrosis (CF) patients and control subjects

\begin{tabular}{lcc}
\hline & CF & Control \\
\hline TST h & $4.7 \pm 1.8^{\#}$ & $5.9 \pm 0.4$ \\
SE \% & $71 \pm 25^{\star}$ & $93 \pm 4$ \\
Sleep latency min & $36.0 \pm 62.5$ & $4.8 \pm 3.5$ \\
WASO min & $69.6 \pm 58.1^{+}$ & $20.4 \pm 13.6$ \\
Stage 1 \% TST & $16.8 \pm 19.1$ & $5.9 \pm 3.8$ \\
Stage 2 \% TST & $46.7 \pm 13.9$ & $52.0 \pm 8.1$ \\
SWS \% TST & $22.7 \pm 9.6$ & $24.2 \pm 0.1$ \\
REM \% TST & $13.8 \pm 6.7$ & $19.5 \pm 4.3$ \\
MA $\mathrm{h}^{-1}$ & $14.3 \pm 12.0$ & $9.3 \pm 4.8$ \\
Awakenings $\cdot \mathrm{h}^{-1}$ & $4.2 \pm 2.7^{\S}$ & $2.4 \pm 1.4$ \\
PLM $^{-1} \mathrm{~h}^{-1}$ & $3.6 \pm 6.6$ & $2.4 \pm 3.5$ \\
\hline
\end{tabular}

Data are presented as mean \pm SD. TST: total sleep time; SE: sleep efficiency; WASO: wakefulness after sleep onset; Stage 1: stage 1 of nonrapid eye movement sleep; Stage 2: stage 2 of nonrapid eye movement sleep; SWS: slow-wave sleep; REM: rapid eye movement sleep; MA: movement arousal; PLM: periodic leg movements. \#: $\mathrm{p}=0.048$; $: \mathrm{p}=0.004$; ${ }^{+}: \mathrm{p}=0.002 ;{ }^{\S}: \mathrm{p}=0.06$ versus control. 
Table 4. - Polysomnographic data for cystic fibrosis (CF) patients and control subjects

\begin{tabular}{lcc}
\hline & $\mathrm{CF}$ & Control \\
\hline $\mathrm{AHI}$ & $1.5 \pm 1.6$ & $3.5 \pm 1.8$ \\
$\mathrm{Sa}_{\mathrm{a}, \mathrm{O}_{2} \%} \%$ & & \\
$\quad \mathrm{TST}$ & $84.4 \pm 6.8^{\#}$ & $94.3 \pm 1.5$ \\
$\quad \mathrm{NREM}$ & $84.6 \pm 6.5^{\#}$ & $94.5 \pm 1.5$ \\
$\quad \mathrm{REM}$ & $83.5 \pm 9.6^{\#}$ & $94.6 \pm 1.3$ \\
$\mathrm{Pt}_{\mathrm{t}, \mathrm{CO}_{2} \text { mmHg }}$ & & \\
$\quad \mathrm{TST}$ & $46.9 \pm 4.8$ & $49.8 \pm 3.2$ \\
$\mathrm{NREM}$ & $45.8 \pm 4.9$ & $49.9 \pm 3.2$ \\
REM & $48.9 \pm 5.9$ & $50.6 \pm 3.7$ \\
\hline
\end{tabular}

Data are presented as mean \pm SD. AHI: apnoea/hypopnoea index; $\mathrm{Sa}_{\mathrm{a}} \mathrm{O}_{2}$ : arterial oxygen saturation; TST: total sleep time; NREM: nonrapid eye movement sleep; REM: rapid

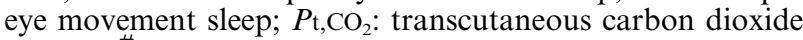
tension. ${ }^{\#}: \mathrm{p}<0.0001$ versus control.

\section{Daytime sleepiness}

The ESS was within normal limits both in $\mathrm{CF}$ patients $(7.3 \pm 4.4)$ and control subjects $(5.6 \pm 4.1)$. The MSLT results are summarized in figure 2. Both groups had short sleep latencies that did not change significantly throughout the day, and overall mean sleep latencies were not significantly different between groups (4.6 $\pm 3 \mathrm{~min}$ in control group versus $6.7 \pm 3 \mathrm{~min}$ in $\mathrm{CF}$ group). Sleep latency was not correlated with daytime oxygenation or other indices of disease severity (BMI, FEV1, 6-min walk distance).

\section{Neurocognitive function}

Subjective Measures (Mood Profile and daytime sleepiness). The significant results of the Mood Profile and the Stanford Sleepiness Scale are shown in figure 3 (mean $\pm \mathrm{SD}$ ) for all four tests during the day, reported on a scale from $1-3$. CF patients reported significantly lower levels of activation $(1.97 \pm 0.03$ versus $2.67 \pm 0.09$;

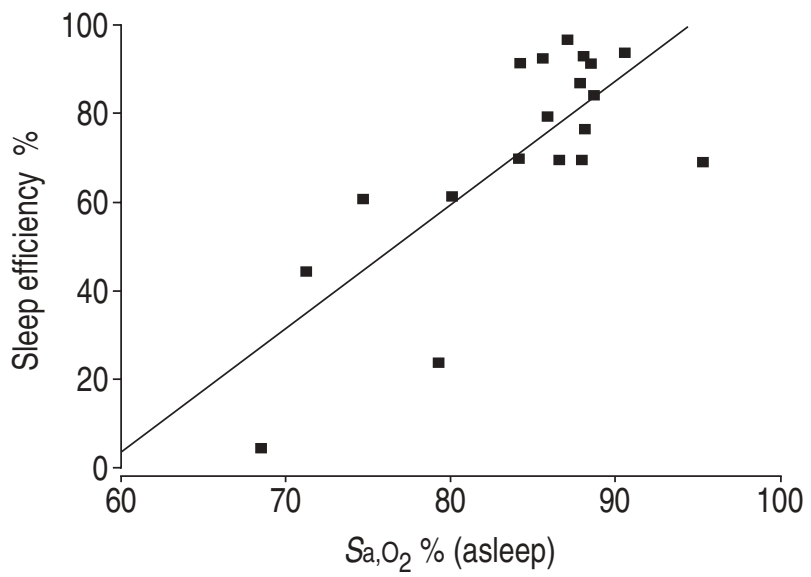

Fig. 1.-Correlation between mean nocturnal oxygen saturation during sleep $\left(\mathrm{S}_{\mathrm{a}} \mathrm{O}_{2}\right)$ and sleep efficiency, during overnight polysomnography. Regression coefficient (represented by solid line)= $0.57 ; \mathrm{p}=0.0014$.

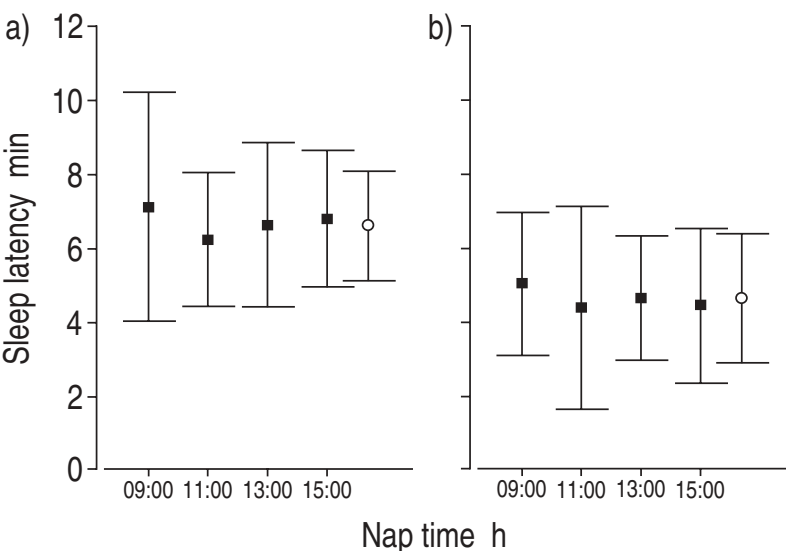

Fig. 2.-Sleep latency in a) cystic fibrosis patients and b) control subjects determined by the multiple sleep latency test. Data are presented as mean \pm SD for each time $(\boldsymbol{a})$ and for total nap time $(\bigcirc)$.

$\mathrm{F}(1,25)=12.45, \mathrm{p}<0.01)$, and happiness $(2.24 \pm 0.02$ versus $2.69 \pm 0.06 ; \mathrm{F}(1,25)=6.32, \mathrm{p}<0.01)$, and a greater level of fatigue $(1.62 \pm 0.10$ versus $1.26 \pm 0.06 ; \mathrm{F}(1,25)=$ $8.25, \mathrm{p}<0.01)$. Although $\mathrm{CF}$ patients reported greater sleepiness on the Stanford Sleepiness Scale than controls, this did not reach statistical significance. Fatigue, activation, happiness and sleepiness did not change significantly across the four test sessions for either the $\mathrm{CF}$ patients or control groups. Scores for depression, anger and fear were not significantly different between the two groups.

In the $\mathrm{CF}$ group, activation was correlated with sleep efficiency (regression coefficient $(\mathrm{r})=0.47, \mathrm{p}=$ 0.05 ), and both activation and happiness correlated with WASO ( $\mathrm{r}=-0.47, \mathrm{p}=0.04 ; \mathrm{r}=-0.55, \mathrm{p}=0.01$, respectively). Activation, happiness, depression and anger were related to the amount of slow wave sleep ( $\mathrm{r}=0.51$, $\mathrm{p}=0.03 ; \mathrm{r}=0.52, \mathrm{p}=0.03 ; \mathrm{r}=-0.55, \mathrm{p}=0.02 ; \mathrm{r}=-0.54$, $\mathrm{p}=0.02$, respectively). Subjective measures of neurocognitive function were not related to hypoxaemia (daytime arterial oxygen tension $\left(\mathrm{Pa}, \mathrm{O}_{2}\right)$, nocturnal $\mathrm{Sa}_{\mathrm{a}} \mathrm{O}_{2}$ ) or other indices of disease severity (BMI,

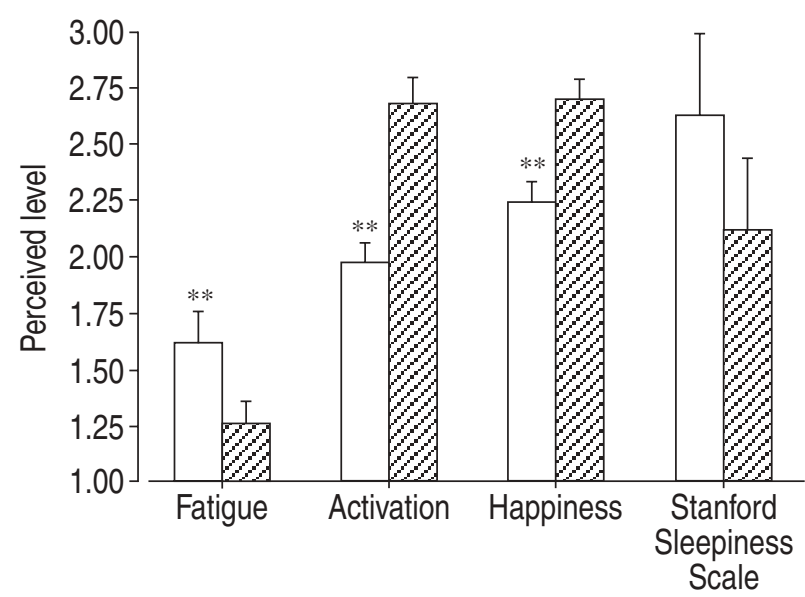

Fig. 3.-Subjective measures of neurocognitive performance in cystic fibrosis (CF) patients and control subjects. Data are represented as mean \pm SD. $\square$ : CF; $\mathbb{Z}$ : control. ${ }^{* *}: \mathrm{p}<0.01$ versus control. 
FEV1). Activation and happiness were not related to sleep latency (MSLT).

Objective performance tasks. The results of the five neurocognitive tasks (mean \pm SD of all four tests across the day) are shown in figure 4 ( $a$ and $b$ ). The CF group had significantly lower throughput (number of correct responses $\left.\cdot \mathrm{min}^{-1}\right)$ for serial reaction $(116.8 \pm 5.7$ versus $162.4 \pm 5.1 ; \mathrm{F}(1,25)=14.28, \mathrm{p}<0.001)$, colour/word conflict $(72 \pm 2.0$ versus $94 \pm 3.1 ; \mathrm{F}(1,25)=10.96, \mathrm{p}<0.001)$ and simple addition/subtraction (28.8 \pm 1.5 versus
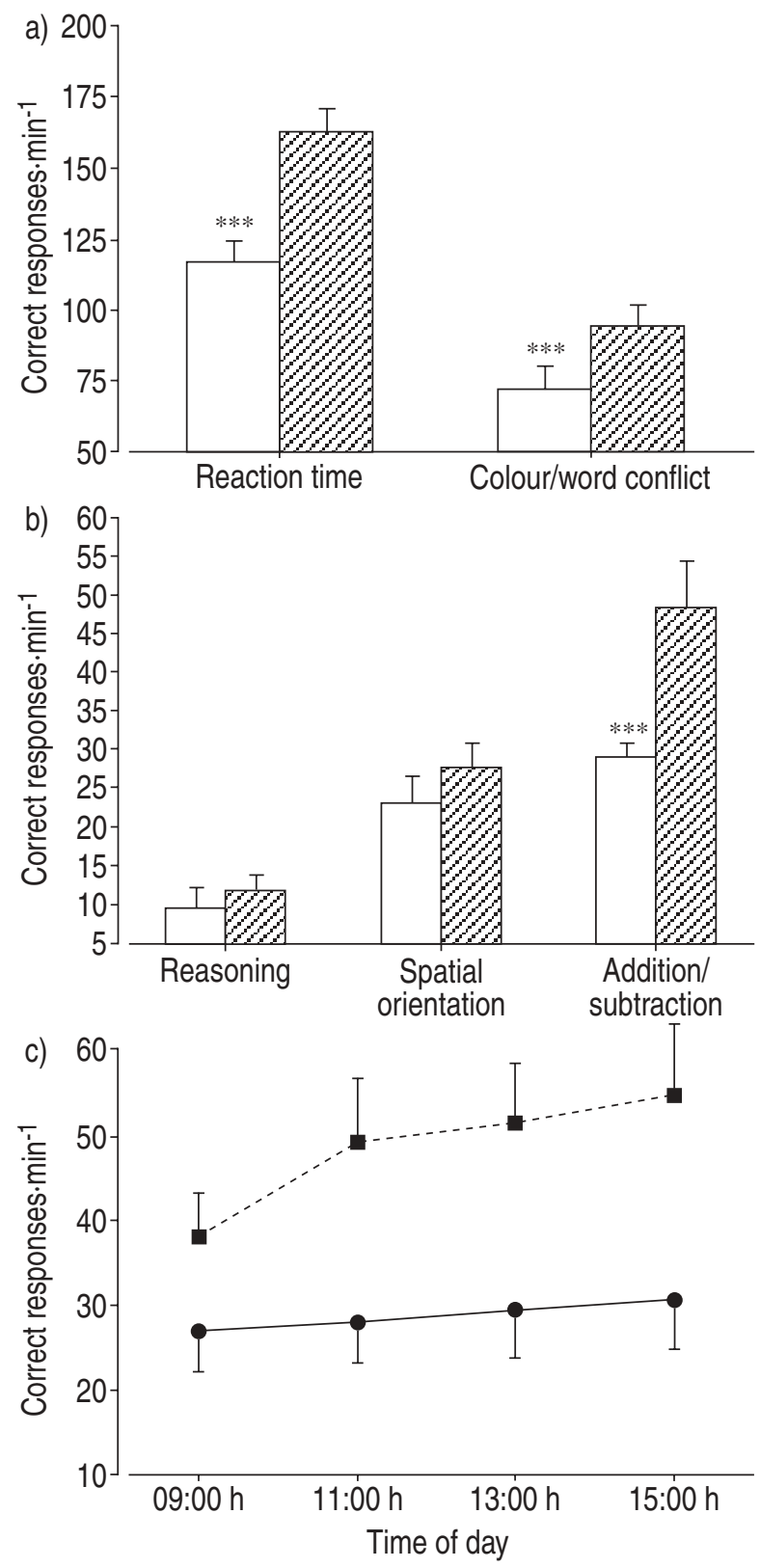

Fig. 4.-Objective assessment of neurocognitive performance in cystic fibrosis (CF) patients $(\square)$ and control subjects $(\mathbb{Z})$. a) Reaction time and colour/word conflict, b) grammatical reasoning, spatial orientation and addition/subtraction and c) the change in addition/subtraction performance for CF $(\mathbf{O})$ and control (a) across the day. Data are presented as mean \pm SD. $* * *: p<0.001$ versus control.
$48.3 \pm 7.1 ; \mathrm{F}(1,25)=11.64, \mathrm{p}<0.01)$ compared to the control group. The change in the simple addition/ subtraction task throughput across the four test sessions is shown in fig. 4c. While the control group showed a marked improvement in their performance across the day on this complex task, the CF group showed only a slight improvement, and the difference in the change between the two groups was statistically significant $(\mathrm{F}(3,75)=5.46, \mathrm{p}<0.001)$.

Serial reaction and colour/word conflict correlated significantly with minimum $\mathrm{Sa}_{\mathrm{a}} \mathrm{O}_{2}$ on the 6-min walk test $(\mathrm{r}=0.59, \mathrm{p}=0.01 ; \mathrm{r}=0.55, \mathrm{p}=0.02$, respectively). No significant correlation was found between any of the objective tests of neurocognitive function and nocturnal or daytime oxygenation. Furthermore, neurocognitive performance was not significantly related to indices of sleep loss, such as sleep efficiency, or with indices of disease severity (BMI, FEV1).

\section{Discussion}

\section{Sleep quality}

Evidence of both sleep disruption and sleep loss in adult $\mathrm{CF}$ patients was found. Potential causes include cough, hypoxaemia, side effects of medication (e.g. $\beta$-agonists), and increased work of breathing. Cough has been noted to disrupt sleep in previous studies of $\mathrm{CF}$ patients [9, 10]. In this study, the technicians reported coughing that interfered with sleep in four patients.

The CF patients had significant daytime hypoxaemia, which worsened during sleep. This may have caused hyperventilation, which could explain the mild reduction in $P \mathrm{t}, \mathrm{CO}_{2}$ in the $\mathrm{CF}$ group. Nocturnal hypoxaemia is well recognized in $\mathrm{CF}$ patients and was an expected finding given our patients' severe airflow limitation [11]. Nocturnal oxygenation was associated with sleep efficiency (fig. 1), which raises the possibility that hypoxaemia may contribute to sleep loss, despite the fact that previous studies of oxygen supplementation in patients with $\mathrm{CF}$ have not demonstrated an improvement in sleep quality $[12,13]$. There is conflicting evidence concerning whether supplemental oxygen therapy improves sleep quality in patients with other forms of chronic lung disease, such as COPD [2, 14, 15].

All of the patients were taking an inhaled $\beta$-agonist, the side effects of which include tachycardia, tremor and excitability. The authors speculate that the use of $\beta$-agonist medication prior to bedtime, which is a ritual for the $\mathrm{CF}$ patients in this study, may account for the prolonged sleep latency observed. Furthermore, this may explain the paradox between prolonged sleep latency at nighttime and short sleep latency on the MSLT. Finally, increased work of breathing associated with severe airflow limitation may also have contributed to the pathogenesis of sleep disruption in these patients. Patients with chronic airflow limitation of the same severity to these patients have increased work of breathing [16] and increasing ventilatory effort is known to be a potent stimulus for arousal from sleep [17]. 


\section{Daytime function}

Both groups were sleepy compared to previously published data in healthy subjects [18] and patients with obstructive sleep apnoea [19]. The control group tended to sleep less (table 2), and may have been more sleep restricted than their diaries suggested. In addition, some members of the control group were familiar with the sleep laboratory environment, which may also have contributed to their surprisingly short sleep latencies. Despite objective evidence of daytime sleepiness, CF patients did not report excessive sleepiness on the ESS. This implies that CF patients may not be aware of excessive sleepiness, which has also been described in other study populations [20].

Cystic fibrosis patients had impaired neurocognitive function, reflected by greater levels of fatigue, lower levels of activation and happiness, and significant deficits in three of the five neurocognitive performance tasks. Overall, adult $\mathrm{CF}$ patients performed at a level of $\sim 60 \%$ of the control group, despite the fact that they had a similar degree of daytime sleepiness. Moreover, neurocognitive performance did not improve over the day in CF patients, in contrast to control subjects (fig. 4c).

\section{Pathogenesis of impaired daytime function}

The pathogenesis of impaired daytime function in adult $\mathrm{CF}$ patients was determined by examining the relationship between two parameters of daytime function (sleepiness and neurocognitive function) and the polysomnographic variables that were significantly different between CF patients and control subjects (oxygenation and sleep loss). Daytime sleepiness, reflected by reduced sleep latency (MSLT), was not significantly related to oxygenation during sleep or to indices of sleep loss such as sleep efficiency. Although there was a trend for an inverse relationship between sleep latency (MSLT) and awake $P \mathrm{a}_{1} \mathrm{O}_{2}$, this did not reach statistical significance $(r=-0.47, p=0.09)$, which may have been partly due to the small patient population. These findings are consistent with those in patients with COPD, where daytime sleepiness has not been found to be related to hypoxaemia [21], but, nevertheless, indicate that nocturnal hypoxaemia promotes sleep loss, reflected by reduced sleep efficiency (fig. 1). Neurocognitive function in CF patients was unrelated to nocturnal or daytime oxygenation. This differs from patients with COPD and chronic respiratory failure, where nocturnal and daytime hypoxaemia have been found to play a significant role in the development of impaired neurocognitive function [22-27]. It is possible that neurocognitive function is less sensitive to hypoxaemia in young adult $\mathrm{CF}$ patients than older patients with COPD. This may also explain why neurocognitive function was not improved by oxygen supplementation in a group of $\mathrm{CF}$ patients comprising mainly children [13].

Conversely, there was an association between sleep loss, reflected by reduced sleep efficiency, and subjective alteration of neurocognitive function, namely reduced activation and happiness, and increased depression and anger. Although the impairment of objective neurocognitive tests in adult CF patients was not clearly related to polysomnographic changes, they were internally consistent with the subjective assessment of fatigue and activation. Similar changes have been reported in association with sleep deprivation in normal subjects [28]. Sleep-deprived individuals also appear to slow their response rate in order to maintain accuracy, which is how the CF patients in this study performed on serial neurocognitive tests (fig. 4c). This suggested that the neurocognitive deficits experienced by the $\mathrm{CF}$ patients may be more related to the long-term impact of chronic sleep deprivation. The finding that the MSLT scores were not significantly different between the control subjects and CF patients supports the notion that the shortterm impact of sleepiness (as noted in the control subjects) is not a likely explanation.

This study has a number of limitations, which should be addressed. Firstly, the sample size was small. Despite the fact that the adult cystic fibrosis clinic is large, the recruitment criteria significantly restricted the number of potentially eligible patients. Secondly, the statistical analyses were limited by the fact that multiple outcome variables were assessed. Thirdly, as already discussed, the control group was sleepier than expected for a healthy population, which hindered some of the comparisons to the cystic fibrosis population. Despite this, significant differences in neurocognitive function were still detected.

\section{References}

1. Fitzsimmons SC. The changing epidemiology of cystic fibrosis. J Pediatr 1993; 122: 1-9.

2. Douglas NJ, Flenley DC. Breathing during sleep in patients with obstructive lung disease. Am Rev Respir Dis 1990; 141: 1055-1070.

3. Bennett LS, Langford BA, Stradling JR, Davies RJO. Sleep fragmentation indices as predictors of daytime sleepiness and nCPAP response in obstructive sleep apnea. Am J Respir Crit Care Med 1998; 158: 778-786.

4. Rechtschaffen A, Kales A. A manual of standardized terminology techniques and scoring system for sleep stages of human sleep. Los Angeles, Brain Information Service/Brain Research Institute, UCLA, 1968.

5. Johns JW. A new method for measuring daytime sleepiness: The Epworth Sleepiness Scale. Sleep 1991; 14: $540-545$.

6. Carskadon MA, Dement WC, Mitler MM, Roth T, Westbrook PR, Keenan S. Guidelines for the Multiple Sleep Latency Test (MSLT): A standard measure of sleepiness. Sleep 1986; 9: 519-524.

7. Thorne DE, Genser SG, Sing HC, Hegge FW. The Walter Reed Performance Battery. Neurobehav Toxicol Teratol 1985; 7: 415-418.

8. Thoracic SA. Standardization of spirometry: 1987 update. Am Rev Respir Dis 1987; 136: 1286.

9. Stokes DC, McBride JT, Wall M, Erba G, Strieder JJ. Sleep hypoxemia in young adults with cystic fibrosis. Am J Dis Child 1980; 134: 741-743.

10. Francis PW, Muller NL, Gurwitz D, Milligan DW. Hemoglobin desaturation: Its occurence during sleep 
in patients with cystic fibrosis. Am J Dis Child 1980; 134: 734-740.

11. Muller NL, Francis PW, Gurwitz D, Levison H, Bryan AC. Mechanism of hemoglobin desaturation during rapid-eye-movement sleep in normal subjects and in patients with cystic fibrosis. Am Rev Respir Dis 1980; 121: 463-469.

12. Spier S, Rivlin J, Hughes D, Levison H. The effect of oxygen on sleep, blood gases, and ventilation in cystic fibrosis. Am Rev Respir Dis 1984; 129: 712-718.

13. Zinman R, Corey M, Coates AL, et al. Nocturnal home oxygen in the treatment of hypoxemic cystic fibrosis patients. J Pediatr 1989; 114: 368-377.

14. Fleetham J, West P, Mezon B, Conway W, Roth T, Kryger M. Sleep, arousals, and oxygen desaturation in chronic obstructive pulmonary disease. Am Rev Respir Dis 1982; 126: 429-433.

15. Calverley PM, Brezinova V, Douglas NJ, Catterall JR, Flenley DC. The effect of oxygenation on sleep quality in chronic bronchitis and emphysema. Am Rev Respir Dis 1982; 126: 206-210.

16. Lanigan C, Moxham J, Ponte J. Effect of chronic airflow limitation on resting oxygen consumption. Thorax 1990; 45: 388-390.

17. Gleeson K, Zwillich CW, White DP. The influence of increasing ventilatory effort on arousal from sleep. $\mathrm{Am}$ Rev Respir Dis 1990; 142: 295-300.

18. Richardson GS, Carskadon MA, Flagg W, van den Hoed J, Dement WC, Mitler MM. Excessive daytime sleepiness in man: Multiple sleep latency measurement in narcoleptic and control subjects. Electroencephalogr Clin Neurophysiol 1978; 45: 621-627.

19. Reynolds CF, Coble PA, Kupfer DJ, Holzer BC. Application of the multiple sleep latency test in disorders of excessive sleepiness. Electroencephalogr Clin Neurophysiol 1982; 55: 443-452.
20. Pilcher JJ, Walters AS. How sleep deprivation affects psychological variables related to college students' cognitive performance. J Am Coll Health 1997; 46: 121-126.

21. Orr WC, Shamma-Othman Z, Levin D, Othman J, Rundell $\mathrm{OH}$. Persistent hypoxemia and excessive daytime sleepiness in chronic obstructive pulmonary disease. Chest 1990; 97: 583-585.

22. Grant I, Heaton RK, McSweeny AJ, Adams KM, Timms RM. Neuropsychological findings in hypoxemic chronic obstructive pulmonary disease. Arch Intern Med 1982; 142: 473-478.

23. Krop HD, Block AJ, Cohen E. Neuropsychological effects of continuous oxygen therapy in chronic obstructive pulmonary disease. Chest 1973; 64: 317322.

24. Block AJ, Castle JR, Keitt AS. Chronic oxygen therapy: Treatment of chronic obstructive pulmonary disease at sea level. Chest 1974; 65: 279-288.

25. Nocturnal Oxygen Therapy Trial Group. Continuous or nocturnal oxygen therapy in hypoxemic chronic obstructive lung disease: A clinical trial. Ann Intern Med 1980; 93: 391-398.

26. Grant I, Heaton RK, McSween AJ, Adams K, Timms R. Brain dysfunction in COPD. Chest 190: 77: 14701476.

27. Heaton RK, Grant I, McSweeny AJ, Adams KM, Petty TL. Psychological effects of continuous and nocturnal oxygen therapy in hypoxemic chronic obstructive pulmonary disease. Arch Intern Med 1983; 143: 1941-1947.

28. Dinges DF, Pack F, Williams K, et al. Cumulative sleepiness, mood disturbance, and psychomotor vigilance performance decrements during a week of sleep restricted to 4-5 hours per night. Sleep 1997; 20: 267270. 